doi: $10.13108 / 2021-13-4-3$

\title{
ON CONDITION OF REPRESENTING A SUBSPACE IN SCHWARTZ SPACE INVARIANT WITH RESPECT TO DIFFERENTIATION AS DIRECT SUM OF ITS RESIDUAL AND EXPONENTIAL COMPONENTS
}

\author{
N.F. ABUZYAROVA
}

\begin{abstract}
In the work we consider the Schwartz space $\mathcal{E}$ of infinitely differentiable functions on the real line and its closed subspaces invariant with respect to the differentiation operator. It is known that each such space possesses, possibly trivial, exponential and residual components, which are defined by a multiple sequence of points $(-\mathrm{i} \Lambda)$ in the complex plane (spectrum $W$ ) and by a relatively closed in $\mathbb{R}$ segment $I_{W}$ (residual interval of the subspace $W$ ).

Recent studies showed that under certain restrictions for the behavior of $\Lambda$ and $I_{W}$, the corresponding invariant subspace $W$ is uniquely recovered by these characteristics, that is, it admits a spectral synthesis in a weak sense. In the case when the spectrum $(-i \Lambda)$ is a finite sequence, the exponential component of the subspace $W$ is finite-dimensional and the subspace $W$ is the algebraic sum of the residual subspace and a finite-dimensional span of the set of exponential monomials contained in $W$. In the case of an infinite discrete spectrum we obtained the conditions, under which the algebraic sum of the residual and exponential subspaces in $W$ is closed, and hence, it is a direct topological sum coinciding with $W$. These conditions were general but not convenient enough for a straightforward checking. Here we obtain transparent easily checked conditions for the infinite sequence $\Lambda$, under which the invariant subspace $W$ with the spectrum $(-\mathrm{i} \Lambda)$ and the residual interval $I_{W}$ is a direct algebraic and topological sum of its exponential and residual components, that is, each element in $W$ is uniquely represented as a sum of two functions, one of which is the limit of a sequence of exponential monomials in $\mathcal{E}$, while the other vanishes identically on $I_{W}$.
\end{abstract}

Keywords: invariant subspace, spectral synthesis, entire function, Schwartz space.

Mathematics Subject Classification: 30D15, 30E5, 42A38

\section{INTRODUCTION}

Let $\mathcal{E}:=C^{\infty}(\mathbb{R})$ be the Schwartz space equipped with its standard metrizable topology, $W$ be its closed subspace invariant with respect to the differentiation operator $D=\frac{\mathrm{d}}{\mathrm{d} t}$, or, shortly, a $D$-invariant subspace.

Assume that the spectrum of restriction $\sigma_{W}$ of the differentiation operator $D: W \rightarrow W$ is discrete and is equal to $(-\mathrm{i} \Lambda)$, where $\Lambda=\left\{\left(\lambda_{j} ; m_{j}\right)\right\}, \lambda_{j} \in \mathbb{C}, m_{j}$ is the multiplicity of a point $\lambda_{j}$. If $\Lambda \neq \emptyset$, then the set $E(W)$ of exponential monomials (root elements of the differentiation operator) contained in $W$ coincides with the set

$$
\left\{t^{k} e^{-\mathrm{i} \lambda_{j} t}, \quad k=0, \ldots, m_{j}-1, \quad j=1,2, \ldots\right\} .
$$

N.F. Abuzyarova, On condition of Representing a Subspace in Schwartz Space invariant With RESPECT TO DIFFERENTIATION AS DIRECT SUM OF ITS RESIDUAL AND EXPONENTIAL COMPONENTS.

(C) Abuzyarova N.F. 2021.

The research is made in the framework of the state task of the Ministry of Education and Science of Russian Federation (code of scientific project FZWU-2020-0027).

Submitted July 24, 2021. 
We note that generally speaking, the spectrum $\sigma_{W}$, and hence, the set $E(W)$, can be empty for a nontrivial $W$, see [1]. In any case, each $D$-invariant subspace $W$ contains (possibly trivial) exponential $D$-invariant subspace $W_{\text {exp }}=\overline{\operatorname{span} E(W)}$.

It was proved in work [1] that each $D$-invariant subspace $W$ also contains a residual $D$ invariant subspace, namely, there exists a minimal for $W$ relatively closed in $\mathbb{R}$ segment $I_{W}$ such that $W_{I_{W}} \subset W$, where

$$
W_{I_{W}}:=\left\{f \in \mathcal{E}: f=0 \text { on } I_{W}\right\} .
$$

The segment $I_{W}$ is called a residual interval of the subspace $W$. It is clear that the residual component $W_{I_{W}}$ of the subspace $W$ can be trivial and this corresponds to the case $I_{W}=\mathbb{R}$.

In [1], the authors formulated a problem: to find out under which conditions a given $W$ with a discrete spectrum is uniquely determined by the set $E(W)$ and the subspace $W_{I_{W}}$ (or, equivalently, by the sequence $\Lambda$ and the segment $I_{W}$ ), that is, the identity holds:

$$
W=\overline{W_{I_{W}}+\operatorname{span} E(W)} ?
$$

In the same work it was established that (1.1) holds if the set $E(W)$ (and hence, a generating it sequence $\Lambda)$ is finite, in particular, is empty. In this case $W_{\text {exp }}=\operatorname{span} E(W)$ and

$$
W=W_{I_{W}}+W_{\text {exp }}
$$

The problem on spectral synthesis (1.1) and other close issues were studied in details for the space $\mathcal{E}$ in works [2]- 9 ]

In particular, in [9] we studied a possibility of strengthening (1.1) up to the representation

$$
W=W_{I_{W}} \oplus W_{\text {exp }}
$$

which generalizes (1.2) for the case of an infinite set $E(W)$. Conditions obtained in [9] are formulated in terms of existence of a slowly decaying (or invertible in Ehrenpreis sense) entire function vanishing on the sequence $\Lambda$.

We recall that a function $\varphi \in \mathcal{P}$ is called slowly decaying if there exists a number $a>0$ with the property

$$
\forall x \in \mathbb{R} \quad \exists x^{\prime} \in \mathbb{R}:\left|x-x^{\prime}\right| \leqslant a \ln (2+|x|) \quad \text { and }\left|\varphi\left(x^{\prime}\right)\right| \geqslant(2+|x|)^{-a} .
$$

In the present note, as an example of applying the results from [9], we provide conditions for the sequence $\Lambda$, under which the synthesized $D$-invariant subspace (1.1) is represented as a direct sum of its residual and exponential subspaces, that is, it is of form (1.3).

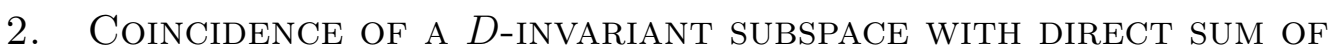 ITS RESIDUAL AND EXPONENTIAL SUBSPACES}

Before we formulate and prove the main result, Theorem 2.1, we introduce some notations and recall some fact we shall need for further arguing.

The union of two disjoint natural subsequences $\mathcal{N}=\left\{n_{k}\right\}$ and $\mathcal{M}=\left\{m_{j}\right\}$ taken in the ascending order is denoted by $\left\{N_{i}\right\}$. Defining on the sets $\mathcal{N}$ and $\mathcal{M}$ real-valued functions

$$
\beta: \mathcal{N} \rightarrow \mathbb{R}, \quad \gamma: \mathcal{M} \rightarrow \mathbb{R},
$$

defines naturally a function $\alpha$ on the sequence $\left\{N_{i}\right\}$ so that its restriction on the set $\mathcal{N}(\mathcal{M})$ coincides with $\beta$ (respectively, with $\gamma$ ). Given a real sequence $\Lambda, 0 \notin \Lambda$, by the symbols $\Lambda^{+}=\left\{\lambda_{k}^{+}\right\}$and $\Lambda^{-}=\left\{\lambda_{j}^{-}\right\}$we denote the subsequences $\Lambda \bigcap(0 ;+\infty)$ and $\Lambda \bigcap(-\infty ; 0)$. And finally, the symbol $D_{B M}(\Lambda)$ stands for a Beurling-Malliavin density of the sequence $\Lambda$, the definition see, for instance, in [10]. 
We recall that by a well-known Beurling-Malliavin theorem, see, for instance, [10], the quantity $\pi D_{B M}(\Lambda)$ coincides with a completeness radius $\rho_{\Lambda}$ of the sequence $\Lambda$. The latter is defined as the infimum of all positive numbers $r$, for which the system of exponential monomials

$$
\left\{t^{k} e^{-\mathrm{i} \lambda_{j} t}, \quad k=0, \ldots, m_{j}-1, \quad j=1,2, \ldots\right\}
$$

is incomplete in $L^{2}(-r ; r)$ and also, what is equivalent, in $C^{\infty}(-r ; r)$.

An important role of the quantity $\rho_{\Lambda}$ in solving spectral synthesis problem (1.1) is due to the validity of the following statement: if $\rho_{\Lambda}<d$, then a $D$-invariant subspace with a discrete spectrum $(-\mathrm{i} \Lambda)$ and a residual interval $[-d ; d]$ is unique and therefore, it is of form $(1.1)$, see [2, Cor. 2], and also [3, Thm. 1.1].

A Fourier-Laplace transform $\mathcal{F}$ acts by the formula

$$
\varphi(z)=\mathcal{F}(S)=S\left(e^{-\mathrm{i} t z}\right), \quad S \in \mathcal{E}^{\prime}
$$

and according to Paley-Wiener-Schwartz theorem, it is a one-to-one correspondence between a strong dual space $\mathcal{E}^{\prime}$ and the space $\mathcal{P}$ of all entire functions of exponential type growing at most polynomially along the real line. With the topology induced by $\mathcal{E}^{\prime}$, the space $\mathcal{P}$ is a topological algebra with respect to the multiplication of the functions; this algebra is called Schwartz algebra. An internal description of the topology in $\mathcal{P}$ is well-known, see, for instance, [2]. We do not reproduce it here since we shall not need it. We only mention that the above said facts imply the following: if for a sequence $\Lambda \subset \mathbb{C}$ the quantity $D_{B M}(\Lambda)$ is finite, then it coincides with the infimum of all positive numbers $c$, for which there exists a function $\varphi \in \mathcal{P}$ of the exponential type $\pi c$ vanishing on $\Lambda$.

In work [9], while studying representation (1.3), for a complex sequence $\Lambda$ with $D_{B M}(\Lambda)<$ $+\infty$ we introduce a new characteristics $D_{s d}(\Lambda)$. Namely, the quantity $D_{s d}(\Lambda)$ is let to be $+\infty$ if $\Lambda$ is not a zero set for any slowly decaying function $\varphi \in \mathcal{P}$. Otherwise $D_{s d}(\Lambda)$ is defined as the infimum of all positive numbers $c$ such that there exists a slowly decaying function $\varphi \in \mathcal{P}$ of the exponential type $\pi c$ vanishing on $\Lambda$.

It is known that the presence of the slow decay property of $\varphi$ is equivalent to the fact the operator of the convolution with the functional $S=\mathcal{F}^{-1}(\varphi)$ acts surjectively from $\mathcal{E}$ into $\mathcal{E}$ [11]. L. Ehrenpreis called such functionals in $\mathcal{E}^{\prime}$ invertible. This explains a term "functions invertible by Ehrenpreis", which we proposed in [12] and which is equivalent to the term "slowly decaying function".

We proceed to the formulation of our main result.

Theorem 2.1. Let a real sequence $\Lambda$ be such that there exist $c_{0}>0$ and natural subsequences $\mathcal{N}=\left\{n_{k}\right\}$ and $\mathcal{M}=\left\{m_{j}\right\}, \mathcal{N} \cap \mathcal{M}=\emptyset$ with a property: functions

$$
\beta: \mathcal{N} \rightarrow \mathbb{R}, \quad \gamma: \mathcal{M} \rightarrow \mathbb{R}
$$

defined by the formulae

$$
\beta\left(n_{k}\right)=\lambda_{k}^{+}-\frac{n_{k}}{c_{0}}, \quad \gamma\left(m_{j}\right)=\lambda_{j}^{-}+\frac{m_{j}}{c_{0}}
$$

are nonnegative and a function $\alpha:\left\{N_{i}\right\} \rightarrow \mathbb{R}$ defined on the union $\left\{N_{i}\right\}$ in the aforementioned way satisfies the condition

$$
\left|\alpha_{j}-\alpha_{i}\right| \leqslant a_{0}\left(\ln ^{2} N_{j}-\ln ^{2} N_{i}\right), \quad j>i,
$$

where $a_{0}$ is a positive constant and we have denoted $\alpha_{i}=\alpha\left(N_{i}\right)$.

Then for each $d_{0}>c_{0}$ there exists a unique $D$-invariant subspace $W$ with a discrete spectrum $\sigma_{W}=(-\mathrm{i} \Lambda)$ and a residual interval $I_{W}=\left[-\pi d_{0} ; \pi d_{0}\right]$, and

$$
W=W_{I_{W}} \oplus W_{\text {exp }}
$$


Proof. Let us construct a continuation of the function $\alpha$ on entire $\mathbb{N}$ keeping property (2.1).

Changing, if it is needed, finitely many terms of the sequence $\Lambda$, we can suppose that $N_{1}=1$. Then it follows from (2.1) that

$$
\alpha_{i} \leqslant a_{0} \ln ^{2} N_{i}, \quad i=2,3, \ldots
$$

We denote $\nu_{i}=a_{0} \ln ^{2} N_{i}-\alpha_{i} \geqslant 0$ and consider natural numbers $n$ located between neighbouring $N_{i}$ and $N_{i+1}$.

A. We first assume that $\alpha_{i+1}-\alpha_{i} \geqslant 0$. Then it follows from 2.1 that $\nu_{i} \leqslant \nu_{i+1}$. If $\nu_{i}=\nu_{i+1}$, then we let

$$
\alpha(n)=a_{0} \ln ^{2} n-\nu_{i}, \quad n=N_{i}+1, \ldots, N_{i+1}-1 .
$$

Otherwise, $\nu_{i+1}>0$ and $\sigma:=\frac{\alpha\left(N_{i+1}\right)}{a_{0} \ln ^{2} N_{i+1}} \in(0 ; 1)$. Let $n_{0}$ be the smallest natural number in the interval $\left(N_{i} ; N_{i+1}\right)$ with the property $a_{0}(1-\sigma) \ln ^{2} n_{0} \geqslant \nu_{i}$. We define a function $\alpha$ for natural $n \in\left(N_{i} ; N_{i+1}\right)$ as follows:

$$
\begin{array}{ll}
\alpha(n)=a_{0} \ln ^{2} n-\nu_{i}, & n=N_{i}+1, \ldots, n_{0}-1, \\
\alpha(n)=\sigma a_{0} \ln ^{2} n, & n=n_{0}, \ldots, N_{i+1}-1 .
\end{array}
$$

It is easy to make sure that

$$
|\alpha(j)-\alpha(i)| \leqslant a_{0}\left(\ln ^{2} j-\ln ^{2} i\right), \quad j \geqslant i, \quad i, j \in\left[N_{i} ; N_{i+1}\right] \cap \mathbb{N} .
$$

B. Now assume that $\alpha_{i+1}-\alpha_{i}<0$. We consider a function

$$
y(t)=\alpha_{i}-a_{0}\left(\ln ^{2} t-\ln ^{2} N_{i}\right)
$$

If $y(n) \geqslant \alpha_{i+1}$ for all $n=N_{i}+1, \ldots, N_{i+1}-1$, then we let

$$
\alpha(n)=y(n), \quad n=N_{i}+1, \ldots, N_{i+1}-1 .
$$

Otherwise, by $n_{0}$ we denote a smallest natural number in the interval $\left(N_{i} ; N_{i+1}\right)$ possessing the property $y\left(n_{0}\right)<\alpha_{i+1}$ and we define

$$
\begin{aligned}
& \alpha(n)=y(n), \quad n=N_{i}+1, \ldots, n_{0}-1, \\
& \alpha(n)=\alpha_{i+1}, \quad n=n_{0}, \ldots, N_{i+1}-1 .
\end{aligned}
$$

As in Case A, it is easy to see that the constructed continuation of the function $\alpha$ satisfies condition $(2.2)$.

Defining $\alpha$ by linearity on each interval $(n ; n+1), n=1,2, \ldots$, we arrive at a function on the ray $[1 ; \infty)$ satisfying the estimate

$$
\left|\alpha\left(t^{\prime \prime}\right)-\alpha\left(t^{\prime}\right)\right| \leqslant b_{0}\left(\ln ^{2} t^{\prime \prime}-\ln ^{2} t^{\prime}\right), \quad 1 \leqslant t^{\prime} \leqslant t^{\prime \prime}<\infty,
$$

where $b_{0}$ is a positive function, generally speaking, not coinciding with $a_{0}$.

We consider a real sequence $\mathcal{Z}=\left\{\zeta_{n}\right\}$, where

$$
\zeta_{n}=\frac{n}{c_{0}}+\alpha(|n|), \quad n= \pm 1, \pm 2, \ldots
$$

It is clear that $\Lambda \subset \mathcal{Z}$. By property 2.3 of the function $\alpha$ and Theorem 1 in work [12] we find that the formula

$$
\varphi(z)=\lim _{R \rightarrow \infty} \prod_{\left|\zeta_{n}\right|<R}\left(1-\frac{z}{\zeta_{n}}\right),
$$

defines an entire function of exponential type $\pi c_{0}$, which is an invertible by Ehrenpreis element of the Schwarz algebra $\mathcal{P}$ vanishing on $\Lambda$. In particular that implies that the completeness radius $\rho_{\Lambda} \leqslant \pi c_{0}$; and hence, according the results of the above cited works [2] and [3], for each $d_{0}>c_{0}$ there exists a unique $D$-invariant subspace $W$ with the discrete spectrum $(-\mathrm{i} \Lambda)$ and the residual interval $I_{W}=\left[-\pi d_{0} ; \pi d_{0}\right]$; this subspace is determined by formula (1.1). 
Since the function $\varphi$ is invertible by Ehrenpreis ("slowly decaying"), then $D_{s d}(\Lambda) \leqslant c_{0}$. Applying Statement a) of Theorem 1 from work [9], we conclude that the subspace $W$ is of form $(1.3)$.

\section{BIBLIOGRAPHY}

1. A. Aleman, B. Korenblum. Derivation-Invariant Subspaces of $C^{\infty} / /$ Comp. Meth. Funct. Theor. 8:2, 493-512 (2008).

2. N.F. Abuzyarova. Spectral synthesis in the Schwartz space of infinitely differentiable functions // Dokl. Akad. Nauk. 457:5, 510-513 (2014). [Dokl. Math. 90:1, 479-482 (2014).]

3. A. Aleman, A. Baranov, Yu. Belov. Subspaces of $C^{\infty}$ invariant under the differentiation// J. Funct. Anal. 268, 2421-2439 (2015).

4. N.F. Abuzyarova. Spectral synthesis for the differentiation operator in the Schwartz space // Matem. Zamet. 102:2, 163-177 (2017) [Math. Notes. 102:2, 137-148 (2017).]

5. N.F. Abuzyarova. Some properties of principal submodules in the module of entire functions of exponential type and polynomial growth on the real axis // Ufimskij Matem. Zhurn. 8:1, 3-14 (2016). [Ufa Math. J. 8:1, 1-12 (2016).]

6. N.F. Abuzyarova. On 2-generateness of weakly localizable submodules in the module of entire functions of exponential type and polynomial growth on the real axis // Ufimskij Matem. Zhurn. 8:3, 8-21 (2016). [Ufa Math. J. 8:3, 8-21 (2016).]

7. N.F. Abuzyarova. Principal submodules in the module of entire functions, which is dual to the Schwarz space, and weak spectral synthesis in the Schwartz space // J. Math. Sci. 241:6, 658-671 (2019).

8. A. Baranov, Yu. Belov. Synthesizable differentiation-invariant subspaces // Geom. Funct. Anal. 29:1, 44-71 (2019).

9. N.F. Abuzyarova. Representation of synthesizable differentiation-invariant subspaces of the Schwartz space // Dokl. RAN. 498:3, 5-9 (2021). [Dokl. Math. 103:3, 99-102 (2021).]

10. P. Koosis. The logarithmic integral. II. Cambridge Univ. Press., Cambridge (1992).

11. L. Ehrenpreis. Solution of some problems of division, IV // Amer. J. Math. 57:1, 522-588 (1960).

12. N.F. Abuzyarova. On shifts of the sequence of integers generating functions that are invertible in the sense of Ehrenpreis // J. Math. Sci. 251:2, 161-175 (2020).

Natalia Fairbakhovna Abuzyarova,

Bashkir State University,

Zaki Validi str. 32,

450076, Ufa, Russia

E-mail: abnatf@gmail.com 